Invited Paper

\title{
High-grade, compact spectrometers for Earth Observation from SmallSats
}

\author{
L.F. van der Wal ${ }^{* a}$, B.T.G. de Goeij ${ }^{\mathrm{a}}$, R. Jansen ${ }^{\mathrm{a}}$, J.A.J. Oosterling ${ }^{\mathrm{a}}$ and B. Snijders ${ }^{\mathrm{a}}$ \\ ${ }^{a}$ TNO, PO Box 155, 2600 AD Delft, The Netherlands
}

\begin{abstract}
The market for nano- and microsatellites is developing rapidly. There is a strong focus on 2D imaging of the Earth's surface, with limited possibilities to obtain spectral information. More demanding applications, such as monitoring trace gases, aerosols or water quality still require advanced imaging instruments, which are large, heavy and expensive. In recent years TNO has investigated and developed different innovative designs to realize advanced spectrometers for space applications in a more compact and cost-effective manner. This offers multiple advantages: a compact instrument can be flown on a much smaller platform (nano- or microsatellite); a low-cost instrument opens up the possibility to fly multiple instruments in a satellite constellation, improving both global coverage and temporal sampling (e.g. to study diurnal processes); a constellation of low-cost instruments may provide added value to the larger scientific and operational satellite missions (e.g. the Copernicus Sentinel missions); and a small, lightweight spectrometer can also be mounted easily on a high-altitude UAV (offering high spatial resolution). Last but not least, a low-cost instrument may allow to break through the 'cost spiral': lower cost will allow to take more risk and thus progress more quickly. This may lead to a much faster development cycle than customary for current Earth Observation instruments. To explore the potential of a constellation of low-cost instruments a consortium of Dutch partners was formed, which currently consists of Airbus Defence \& Space Netherlands, ISISpace, S\&T and TNO. In this paper we will illustrate this new design approach by using the most advanced design of a hyperspectral imaging spectrometer (named 'Spectrolite') as an example. We will discuss the different design and manufacturing techniques that were used to realize this compact and low-cost design. Laboratory tests as well as the first preliminary results of airborne measurements with the Spectrolite breadboard will be presented and discussed. The design of Spectrolite offers the flexibility to tune its performance (spectral range, spectral resolution) to a specific application. Thus, based on the same basic system design, Spectrolite offers a range of applications to different clients. To illustrate this, we will present a mission concept to $\mathrm{monitor}^{\mathrm{NO}_{2}}$ concentrations over urban areas at high spatial resolution, based on a constellation of small satellites.
\end{abstract}

\section{A GROWING MARKET FOR NANO- AND MICROSATELLITES}

The market for nano- and microsatellites is developing rapidly, largely driven by the continued interest of the commercial sector in nano- and microsatellite applications. The following observations are taken from the SpaceWorks 2016 Nano/Microsatellite Market Forecast ${ }^{1}$ :

- Projections based on announced and future plans of developers and programs indicate as many as 3,000 nano/microsatellites will require a launch from 2016 through 2022;

- The commercial sector will contribute over $70 \%$ of future nano/microsatellites;

- The commercial sector will increase its proportional representation over the next three years, to the extent that it will soon account for the majority of spacecraft launched in the $1-50 \mathrm{~kg}$ class;

- In that same period more than $70 \%$ of future nano/microsatellites will be used for Earth Observation and Remote Sensing purposes (compared to $37 \%$ from 2009 to 2015; see Figure 1));

- And over $60 \%$ of future nanosatellites $(1-10 \mathrm{~kg})$ will be in the increasingly popular $4-6 \mathrm{~kg}$ mass class (compared to only 23\% from 2009 to 2013).

*len.vanderwal@tno.nl; phone: +31 888666526,www.tno.nl.

Remote Sensing Technologies and Applications in Urban Environments, edited by Thilo Erbertseder, Thomas Esch, Nektarios Chrysoulakis, Proc. of SPIE Vol. 10008, 1000806

(C) 2016 SPIE · CCC code: 0277-786X/16/\$18 - doi: 10.1117/12.2247299 


\section{Nano/Microsatellite Trends by Purpose (1 - $50 \mathrm{~kg})$}

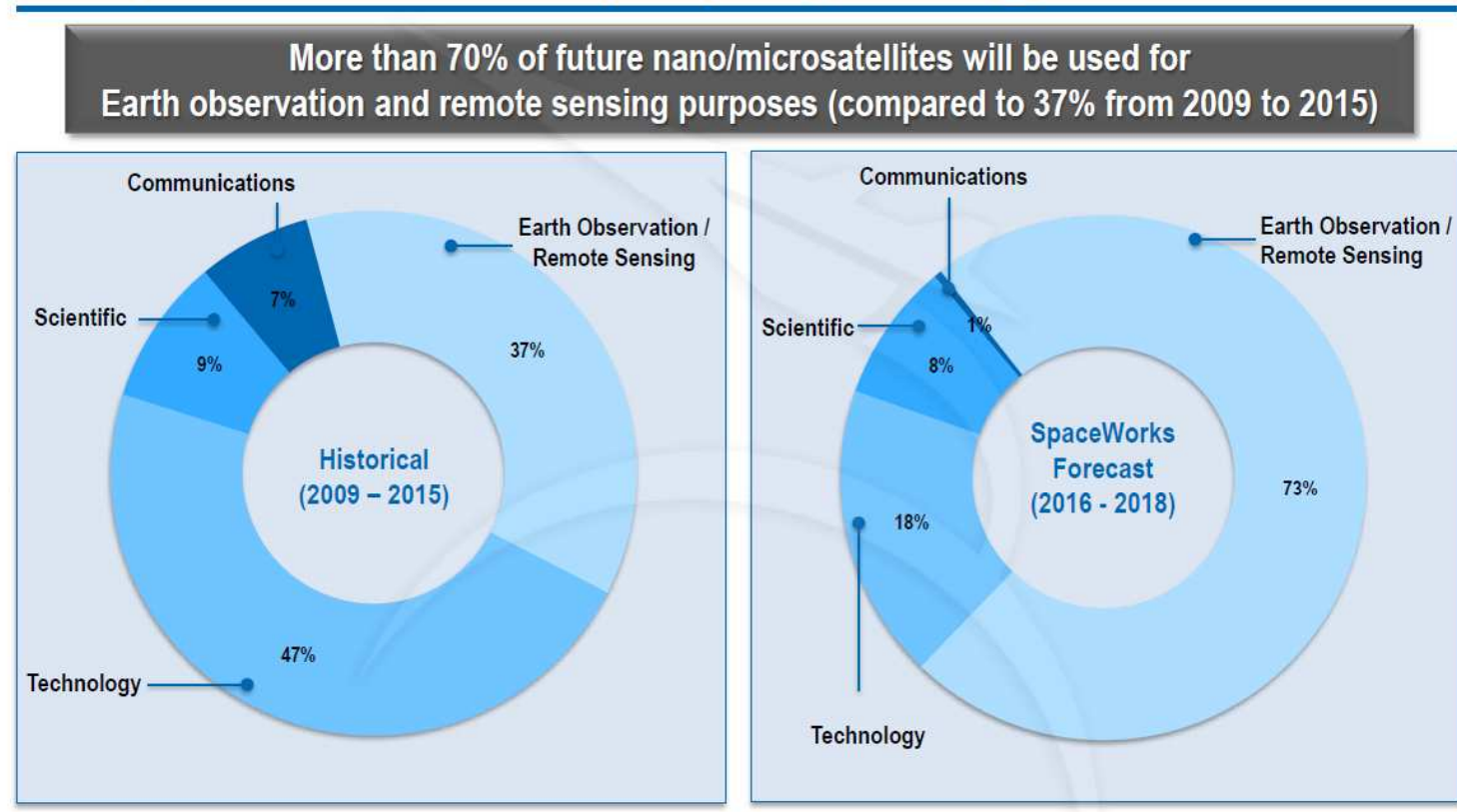

As key companies move toward operations, a smaller proportion of technology development/demonstration nano/microsatellites will be built in the next few years

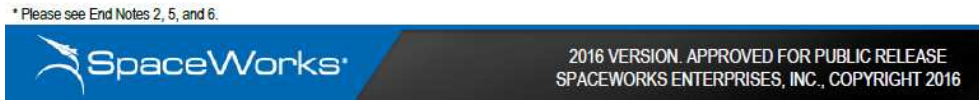

Figure 1. Graphs illustrating the strong increase in Earth Observation and Remote Sensing applications in the nano- and microsatellite market (from the SpaceWorks 2016 Nano/Microsatellite Market Forecast ${ }^{1}$ ).

\section{OBTAINING SPECTRAL INFORMATION}

However, the strong increase in Earth Observation applications is mainly focused on imaging the Earth's surface with medium or high resolution. Possibilities to obtain spectral information are still limited. Therefore, more demanding applications, such as monitoring trace gases and aerosols, or water quality still require advanced spectral imaging instruments, which are large, heavy and expensive. Given our strong background in the design of high-grade atmospheric monitoring instruments, we were challenged to see if we could create a break-through in this area.

In 2013 we started to investigate different innovative designs to realize advanced spectrometers for space applications in a very compact and cost-effective manner. Our R\&D efforts were motivated by the following thoughts:

- A compact instrument can be flown on a much smaller platform (nano- or microsatellite);

- A low-cost instrument opens up the possibility to fly multiple instruments in a satellite constellation, improving both global coverage and temporal sampling (e.g. multiple overpasses per day to study diurnal processes);

- In this way a constellation of low-cost instruments may provide added value to the larger scientific and operational satellite missions (e.g. the Copernicus Sentinel missions);

- A low-cost instrument may also allow us to break through the 'cost spiral': lower cost will allow us to take more risk and thus progress more rapidly. This may lead to a much faster development cycle than customary for current Earth Observation instruments. 


\section{SPECTROLITE}

In this paper we will illustrate the above considerations using the most mature design of a hyperspectral imaging spectrometer (recently named 'Spectrolite') as an example. During the conceptual development of Spectrolite we applied the following principles:

- Make a self-standing system that is optimized for a specific task (i.e. a single observable phenomenon); this keeps the development complexity relatively small and ensures that tough requirements for different observable phenomena are not mingled, keeping the cost for development and production low.

- Make a modular system that can easily be adopted for different observable phenomena (e.g. air quality, land use or water quality) without a full redevelopment and qualification; this should allow the system to be built in small series, allowing the development cost to be shared over multiple modules.

Based on these constraints Spectrolite ended up as an all-reflective, off-axis design, consisting of only 8 optical components, including 4 free-form mirrors and a flat grating in the spectrometer. The all-reflective approach has a number of advantages:

- A favourable thermal stability due to the all-aluminium design (important for a low-mass satellite);

- The design can be copied unchanged for other wavelength ranges between $270 \mathrm{~nm}$ and $2400 \mathrm{~nm}$ (with the exception of the grating and possibly the detector);

- Fewer optical elements due to the insensitivity to chromatic aberrations and the application of diamond turned freeform optics;

- More design freedom regarding stray light baffling due to the off-axis nature of such a design;

- Inherently low ghost stray light between detector and optical surfaces.

\subsection{Spectrolite optical design philosophy}

The design was made in a semi-automated ('brute force') manner. The starting point was found using YYBAR diagrams and SEIDEL aberrations. From this starting point, 10 million design variations were explored by 'brute force' calculations. These 10 million variations were then filtered on parameters like volume, optical path length etc. Finally the designer chose the most promising/optimal solution for further processing. The optimum solution was the input for a CodeV model, which was allowed to optimize further using a number of geometrical constraints (for more details, $\operatorname{see}^{2}$ ). The use of freeform mirrors offers much greater optical design freedom. In traditional freeform systems, this advantage is typically used to boost the optical performance of the system. For Spectrolite it was used to increase the robustness of the design, creating a very stable instrument that can be quickly assembled on manufacturing tolerances. The nominal RMS spot size of the design is $\sim 6.5$ micron. Including quite large tolerances it is $\sim 20$ micron, which is sufficient for its intended purpose.

\subsection{Spectrolite breadboard}

In order to demonstrate that this design philosophy actually worked, a Spectrolite breadboard was developed and built. The breadboard was optimized for the detection of $\mathrm{NO}_{2}$ concentrations in the atmosphere.

For this breadboard the following requirements were set:

- Spectral range: $320 \mathrm{~nm}-495 \mathrm{~nm}$

- Spectral resolution: $<0.5 \mathrm{~nm}$

- Spatial resolution: $0.1^{\circ}(1 \mathrm{~km}$ GSD @ $600 \mathrm{~km})$

- Field of view: $60^{\circ}(\sim 700 \mathrm{~km}$ swath @ $600 \mathrm{~km})$

- SNR for $\mathrm{NO}_{2}$ measurement: 700 over urban areas

Figure 2 shows a 3D drawing of the designed Spectrolite breadboard, including the optical path. 


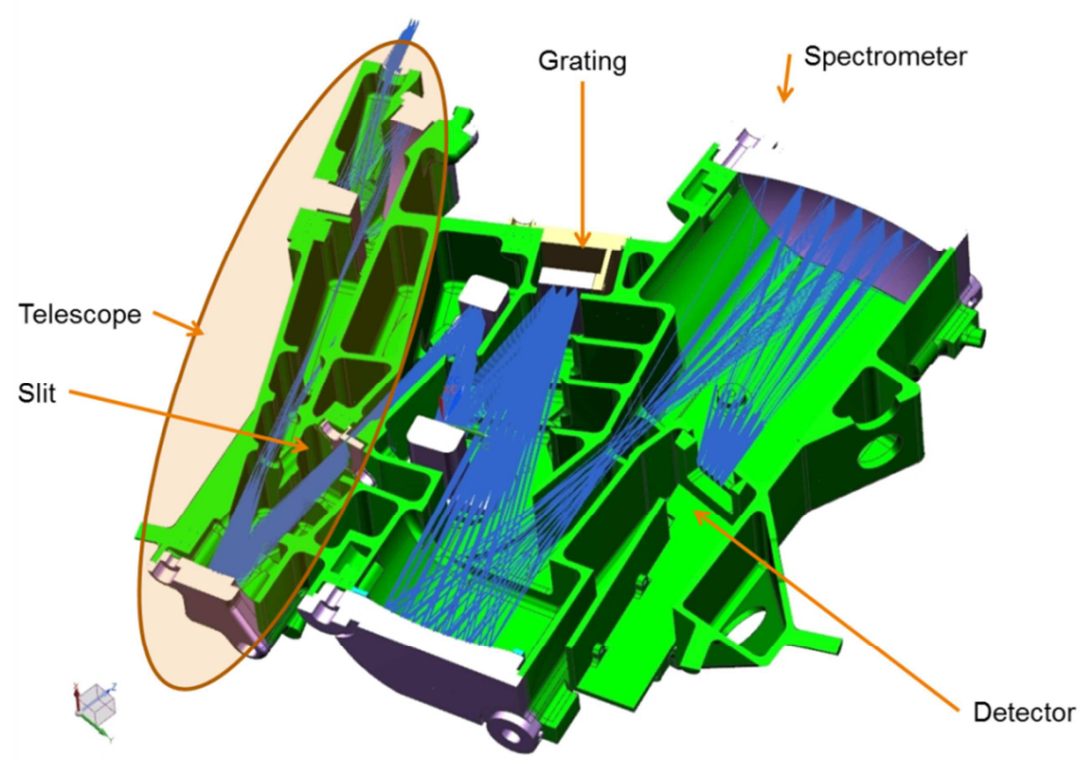

Figure 2. 3D drawing of Spectrolite, showing the housing (green), all components (white) and the optical path (blue).

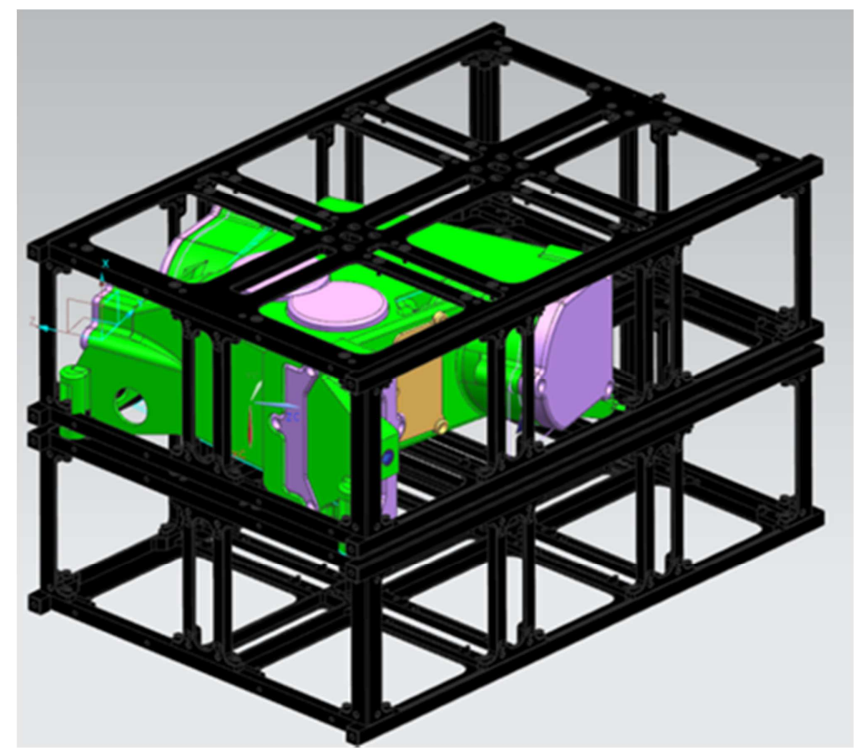

Figure 3. Schematic, showing how Spectrolite fits into the standardized mechanical structure of a large CubeSat.

The design is very compact and fits in a volume of $10 \times 20 \times 20 \mathrm{~cm}^{3}$. As shown in Figure 3, due to this small volume, Spectrolite easily fits in a standardized $6 \mathrm{U}$ or $12 \mathrm{U}$ volume CubeSat structure, while leaving sufficient room for an additional instrument and the supporting hardware (electronics, communication, attitude and thermal control, etc.). Due to financial constraints, only the spectrometer part of Spectrolite was actually realized, leaving the system without a telescope, but with the potential to demonstrate the performance of this all-reflective spectrometer, built in an aluminium housing. 


\section{MANUFACTURING AND ASSEMBLY OF THE SPECTROLITE BREADBOARD}

The Spectrolite housing was manufactured using a relatively novel method for space hardware: 3D printing and investment casting. First, a 3D wax model was printed, from which a ceramic cast was made. Using this cast, three aluminium housings were made (see Section 4.1 for details).

The spatial accuracy of this process is in the order of $0.1 \mathrm{~mm}$, which is insufficient if one wants to mount the mirrors directly without alignment. Therefore the mirror interfaces were post machined to an accuracy of 20 micron.

The four mirrors of the spectrometer (see Figure 4) were manufactured at TNO using Single Point Diamond Turning with a slow servo tool. After this the mirrors were lightly hand polished only. The manufacturing turned out to be more challenging than e.g. the mirrors for Sentinel 5P (Tropomi), due to the more difficult mounting and the 'aggressive' shape with large slopes. This required a very low turning speed, which in turn required very high thermal stability of the cutting tool ( $<10 \mathrm{~nm}$ over a period of minutes or more). Also the effective aperture was only $\sim 1 \mathrm{~mm}$ smaller than the edge of the mirrors. The grating was a COTS item procured from Jobin Yvon.

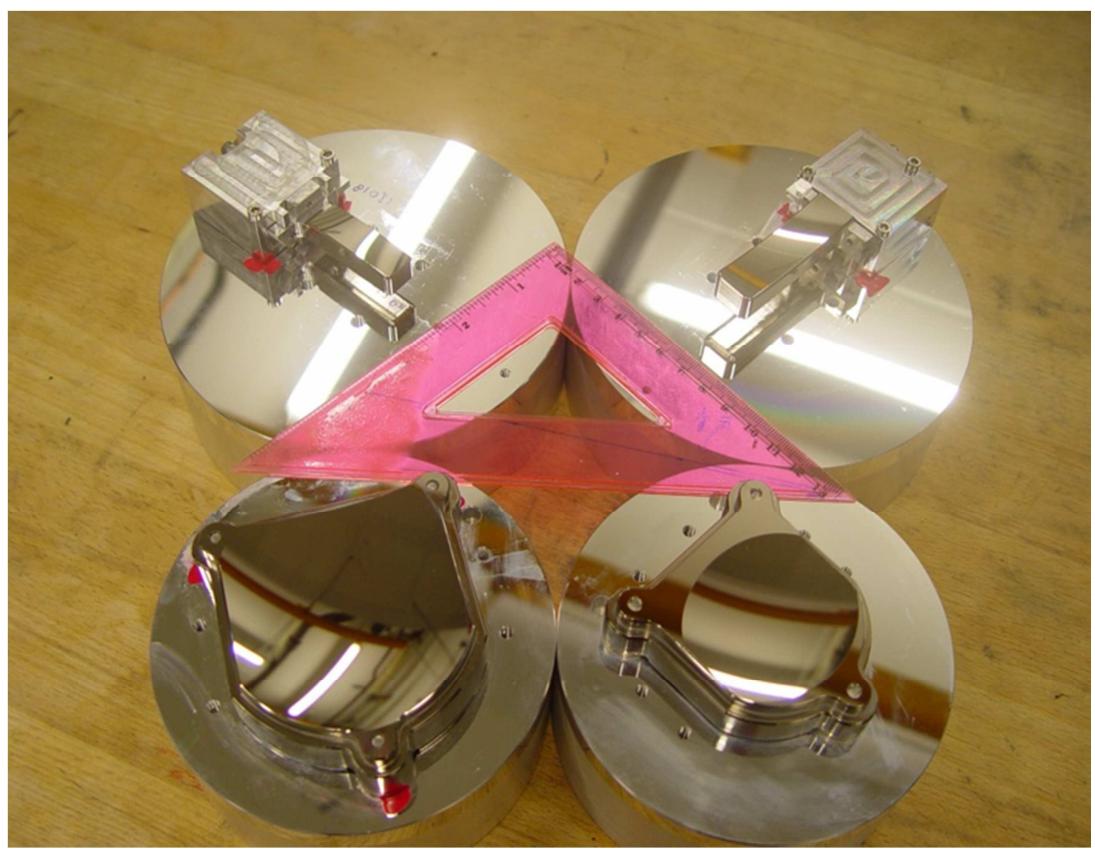

Figure 4. The four freeform mirrors of Spectrolite after manufacturing.

The mirrors and the grating were assembled on manufacturing tolerance, which took approximately 1.5 days. The detector was mounted on shims. Its alignment was checked using a measurement of a spectral line source, as discussed in the verification section (Section 5.1). The measurement was repeated using a number of different shims with varying thickness. The results were fed back into the optical model to predict the position of the ideal focal plane. Finally, the detector was positioned on this plane using 'ideal' shims. The whole alignment of the detector was done in approximately one day. 


\subsection{Additive manufacturing of the housing}

The development of space instruments is mainly influenced by two different drivers:

1. The performance drivers, aiming for:

- An increase in performance of the instrument;

- Higher accuracies of the production process;

- The use of new and better materials.

2. The economic drivers, aiming for:

- Lightweight construction, reducing mass and thus launch costs;

- Cheaper manufacturing processes;

- Shorter development times.

With regard to instrument housings, milling from stock has been the preferred technology to meet the performance and accuracy requirements of space instruments for a very long time. However, emerging technologies, especially additive manufacturing, offer interesting opportunities with regard to many of the above issues, e.g. design freedom and complex shaping (to increase performance), mass and volume reduction (to reduce launch cost), cost-effective manufacturing of small series and decreasing development time.

Given these developments we were interested to find out whether we could use additive manufacturing to realize a highgrade, cost-effective instrument housing for Spectrolite. We selected the combination 3D printing + investment casting*. At this moment, this technology is developed much further than Selective Laser Melting (SLM).

The CAD drawing in Figure 5 shows the housing of Spectrolite, illustrating its complexity. To describe the entire process to manufacture this complex structure by means of investment casting falls outside the scope of this paper. Detailed information on all the process steps that have to be taken can be found in ${ }^{3}$. Here, only some of the most relevant aspects are highlighted.

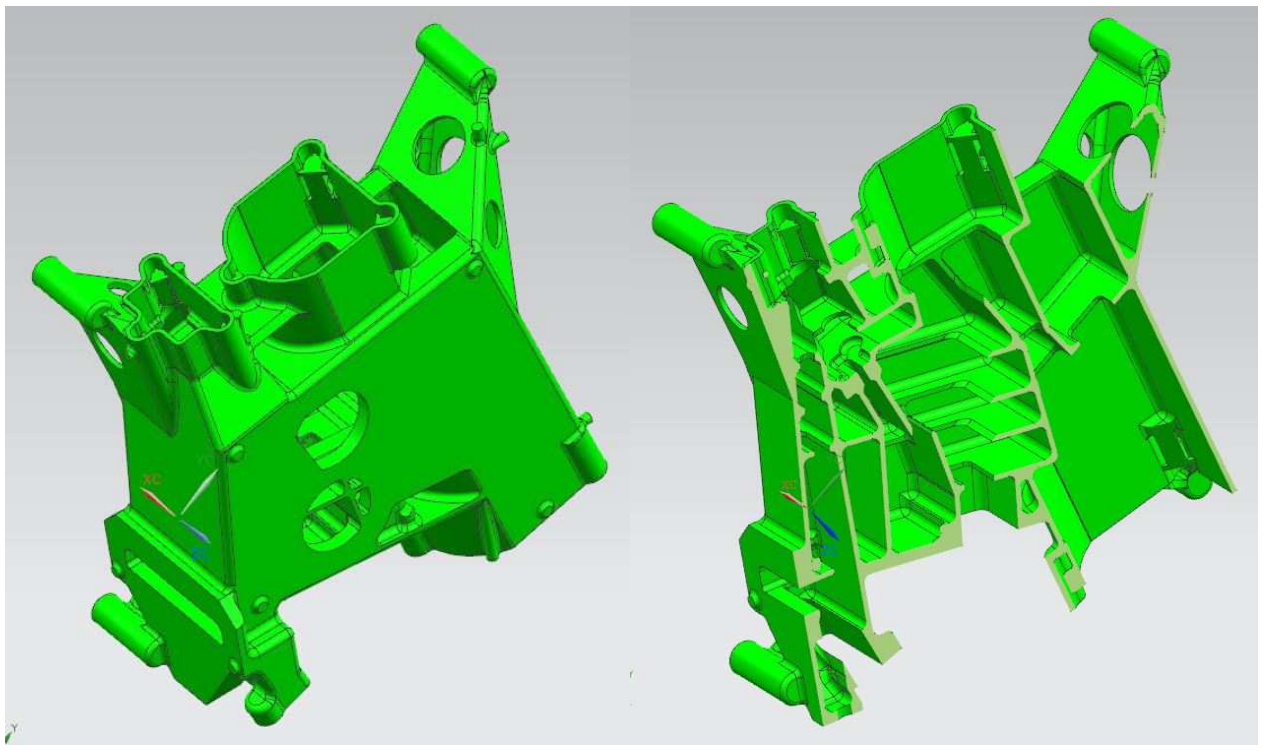

Figure 5. CAD drawing of the Spectrolite housing.

\footnotetext{
* This part of the work has been supported by ESA, as part of the project 'Advanced Manufacturing Methods for Systems-of-Microsystems Nanospacecraft'.
} 


\subsubsection{Additive manufacturing of the housing in wax}

A STEP model of the housing is used to make a wax model by additive manufacturing (as shown in Figure 6). It is this step that provides us with a very large design freedom, as there are hardly any limits to the complexity of the structure to be cast as long as the possibility of post-machining is taken into account carefully.

The wax model is then used to produce an ceramic shell by repeatedly dipping the wax cluster into a ceramic slurry, followed by stucco sanding and drying of each ceramic layer. The ceramic shell is placed in a pressurized oven and heated so the wax flows out of the shell. After dewaxing the ceramic shell is burnt at approximately $2000^{\circ}$ Celsius to get the necessary strength and temperature resistance for the casting process.

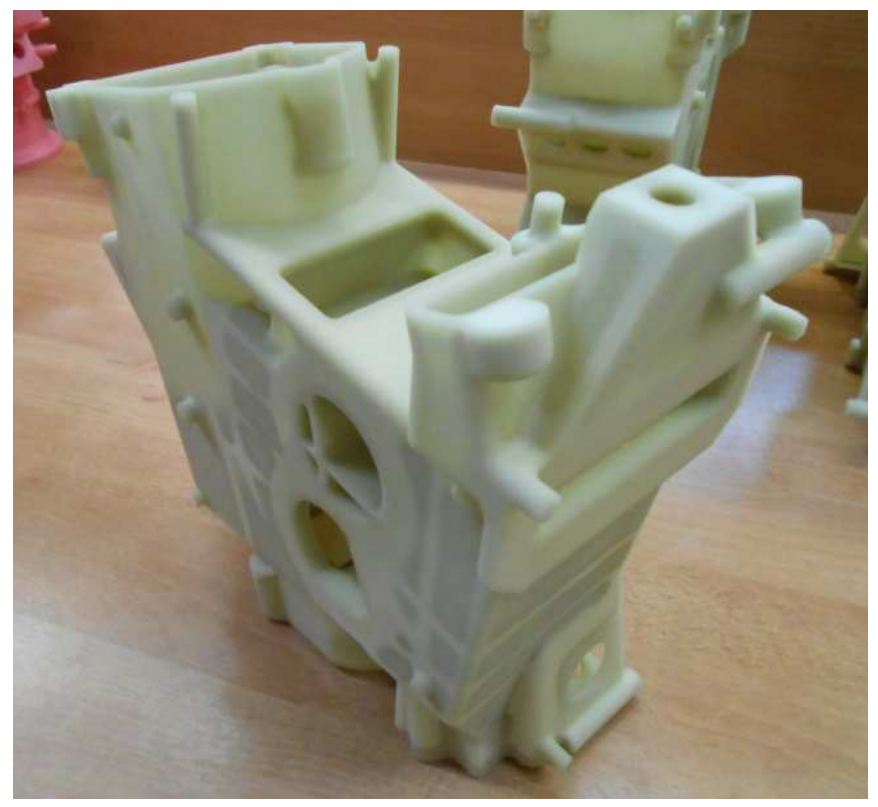

Figure 6. Wax model of the housing made by additive manufacturing.

\subsubsection{Casting of the housing}

For the casting of the housing, we have chosen aluminium alloy A357 (EN AC 42200), because of its excellent casting characteristics, its strength and its welding characteristics. These casting characteristics enable a minimum wall thickness of ca. $2 \mathrm{~mm}$. Possible flaws in the casting can be easily repaired by grinding and welding the bad spots.

The casting is cooled down in a well-controlled manner in a pressurized vessel in a liquid medium to avoid the occurrence of shrinking holes. This also enables the casting of parts with large differences in wall thickness. After the cooling process is finished, the ceramic shell is removed by blasting with water at high pressure. Then the casting is ready for quality inspection (e.g. crack detection, radioscopy and welding repair).

\subsubsection{Heat treatment}

After the quality check the casting is heat-treated in two steps. 'solution heat treatment' followed by 'artificially aging'. Solution heat treatment takes place between $525^{\circ}-545^{\circ}$ Celsius for 12 to 20 hours. Artificially aging is performed at temperature between $150^{\circ}-170^{\circ}$ Celsius for $6-12$ hours. After this heat treatment the casting reaches its final mechanical properties. 


\subsubsection{Milling of all interface surfaces}

All interface surfaces for mounting optical components are milled (finishing). After milling the housing is black anodized (see Figure 7).
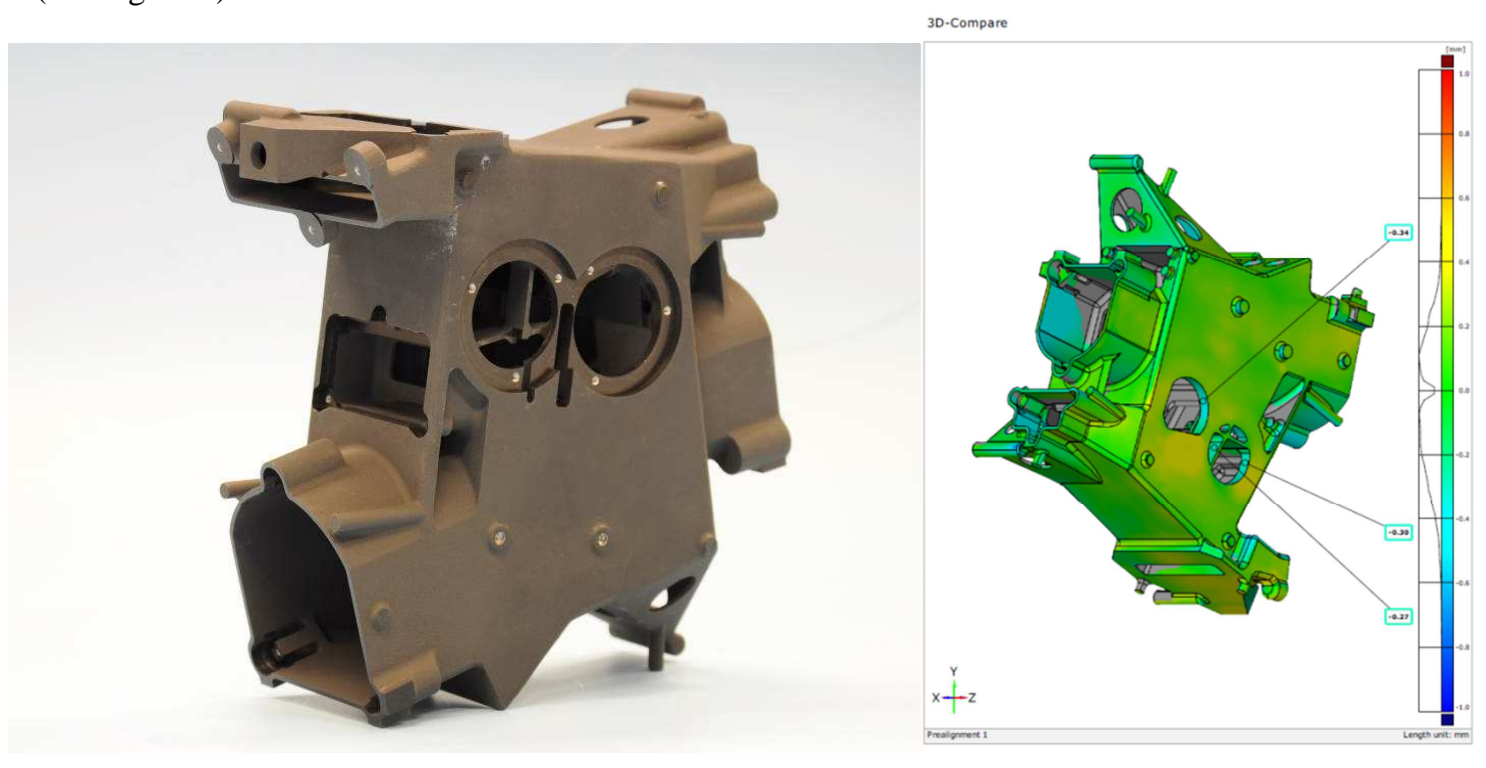

Figure 7. Spectrolite housing finished milled and black anodized (left) and 'best fit' of the optical scan of the housing, indicating the difference between casting and CAD model (right).

\subsubsection{Optical scan}

To increase the efficiency of the finish milling an optical scan of the casting is performed. This will allow the milling company to position the housing in such a way that all interface surfaces that have to be machined, can actually be reached. The optical scan shows that the casting is well within specification (see Figure 7). The requirement on dimensional tolerance was $\pm 0.55 \mathrm{~mm}$. The largest measured deviation between casting and CAD model amounted to $0.51 \mathrm{~mm}$. Most of the deviations were between $-0.30 \mathrm{~mm}$ and $+0.30 \mathrm{~mm}$.

\subsubsection{Cost comparison}

We performed a short cost evaluation of the investment casting process and compared that with the estimated cost of a traditional milling process for different number of housings, as shown in the table below.

Table 1. Cost comparison between '3D printing + casting' and traditional milling

\begin{tabular}{|c|c|}
\hline $\begin{array}{c}\text { Number of } \\
\text { housings }\end{array}$ & Cost difference \\
\hline 1 & $+3 \%$ \\
\hline 4 & $-11 \%$ \\
\hline 10 & $-21 \%$ \\
\hline
\end{tabular}

From this table it is clear that the cost of both manufacturing technologies is about the same for a single housing. For larger series the casting process gradually becomes more cost-effective. 


\section{VERIFICATION OF THE SPECTROLITE BREADBOARD}

A number of tests and analyses have been performed to verify the performance of the spectrometer part of the Spectrolite breadboard. The main performance parameters that were verified were:

- $\quad$ The (RMS) spatial spot size

- The (RMS) spectral spot size

- The polarization sensitivity

- Co-registration

A detailed description of these verification activities falls without the scope of this paper. Here we only present the results of the spectral spot size verification.

\subsection{Spectral spot size verification}

The spectral spot size was measured using a $\mathrm{HgAr}$ spectral line source coupled into a small integrating sphere. The sphere was placed in front of the 'telescope' objective, so that the slit is overfilled in spectral direction, but it was not possible to illuminate the entire slit length. Therefore a number of measurements were performed at several spatial slit positions. Figure 8 shows a single detector frame from the spectral measurements. The three lines correspond to 365, 405 and $436 \mathrm{~nm}$ from top to bottom. A sub frame around each spectral line was selected and the position and height of the peak in each column (i.e. spatial position) was determined. With the results, the 'smile' was removed and the peak heights were normalized, resulting in the plots shown in the left side of Figure 9. For each column, a Gaussian fit was performed, which are shown in the right plots in Figure 9. From the fitted distributions and the known dispersion of the system, the RMS spot size in spectral direction was determined for each column.

This process was repeated for a number of slit positions in order to cover the entire slit. Taking the best result from each measurement, the spectral resolution over the entire slit is plotted in Figure 10. The spectral resolution varies between $\sim 0.2 \mathrm{~nm}$ and $\sim 0.33 \mathrm{~nm}$ and is best for the highest wavelengths. The small gradient in spatial direction might be caused by a non-optimal alignment of the detector. Note that the sharp increase on the right is caused by a measurement artefact; there was no measurement with sufficient SNR for these pixels. The spectral resolution measurement was confirmed by looking closely at the $365 \mathrm{~nm}$ line. In reality this is a double line, with a spacing of $0.47 \mathrm{~nm}$. This doublet could be clearly resolved by the spectrometer, as shown in Figure 11.

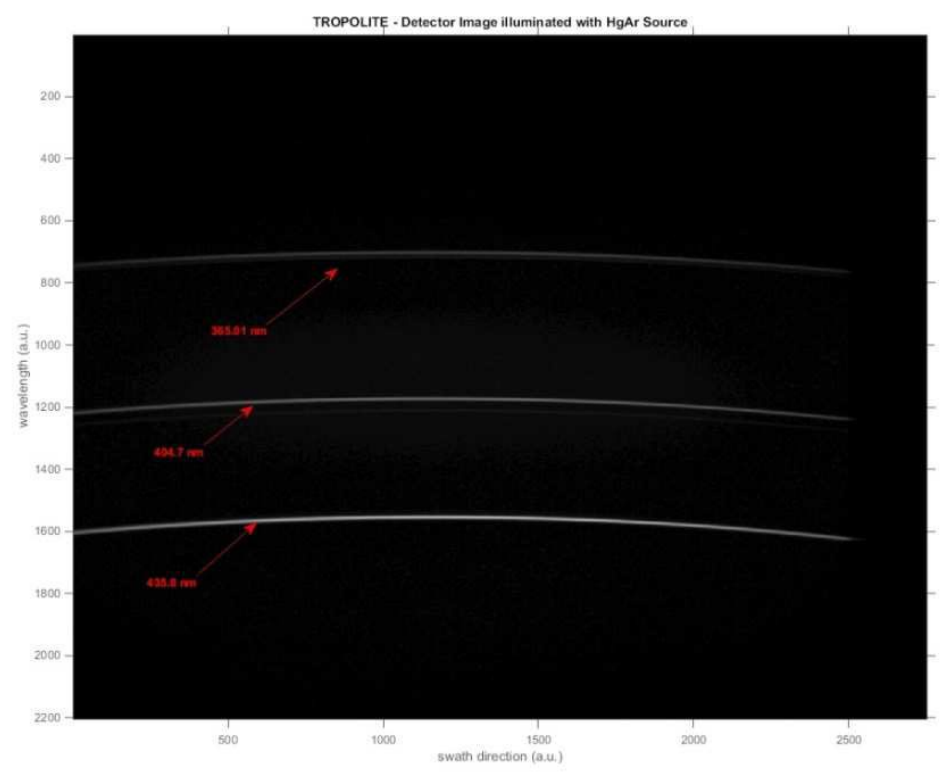

Figure 8. Detector frame; the three lines correspond to 365, 405 and $436 \mathrm{~nm}$ (from top to bottom). 
Sub-frames corrected for smile distortion
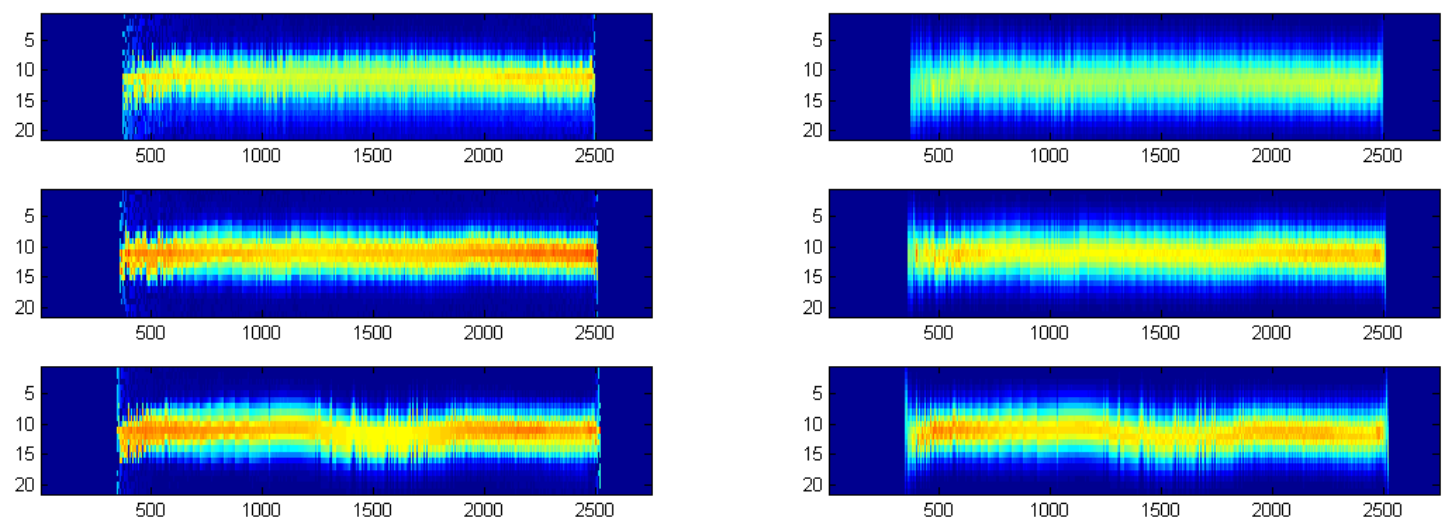

Figure 9. Images of each spectral line (365, 405 and $436 \mathrm{~nm}$ from top to bottom), normalized and with the smile removed (left) and Gaussian fit of the same plots (right).

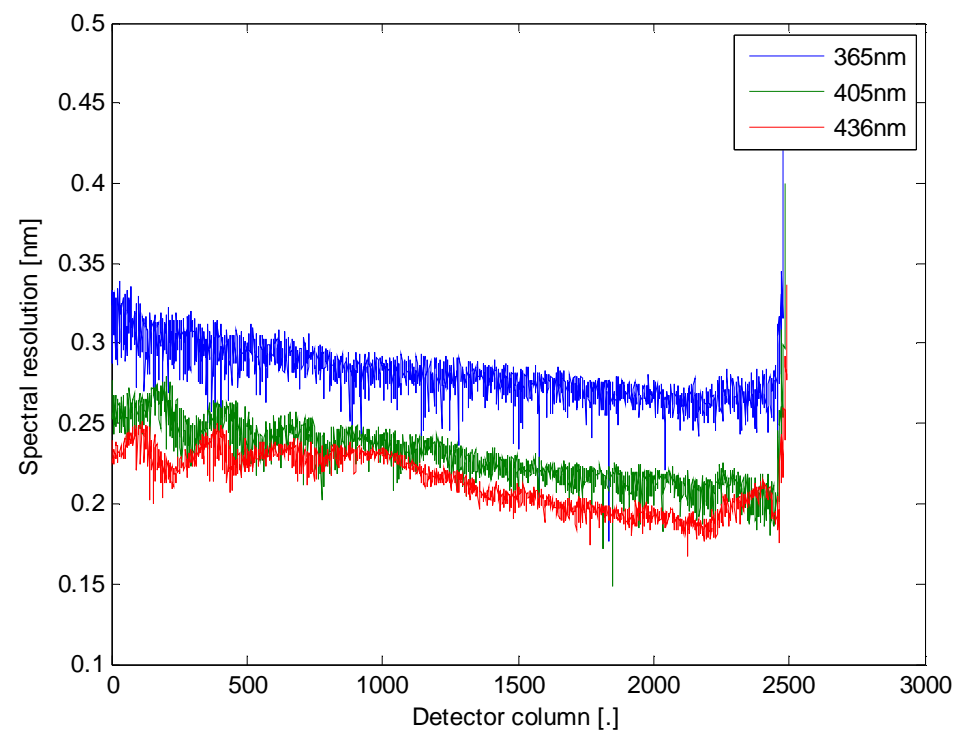

Figure 10. Spectral resolution over the entire slit.
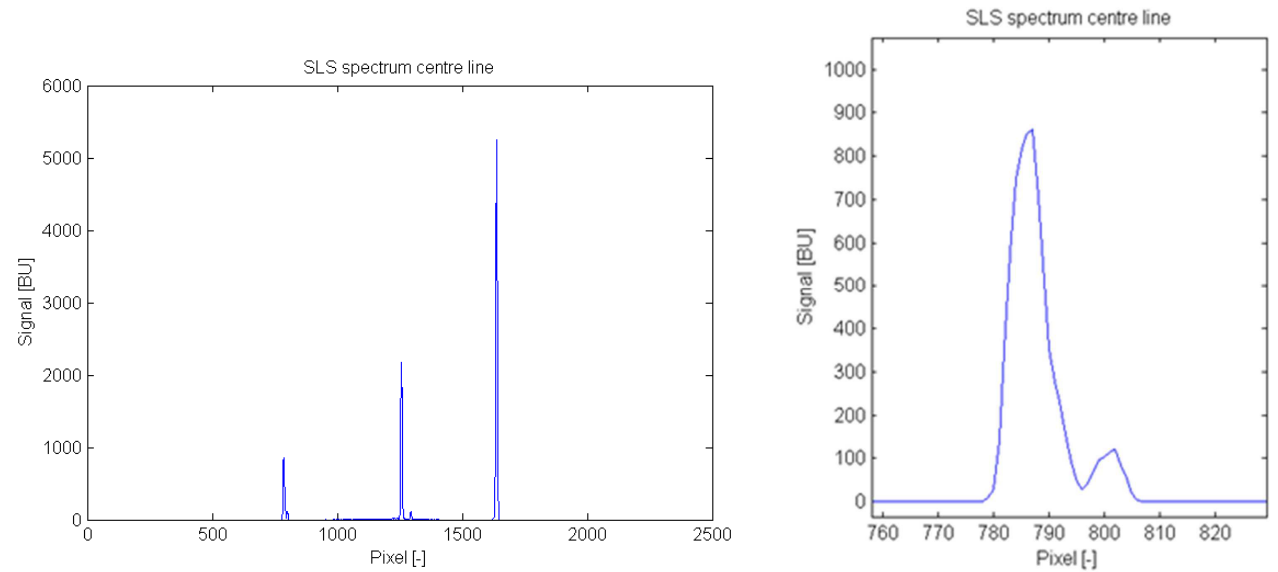

Figure 11. Cross cut through the $\mathrm{HgAr}$ source spectrum (left) and zoom in on the double peak at $365 \mathrm{~nm}$ (right). 


\section{SPECTROLITE CONSORTIUM}

Initiated by the positive results of our efforts to design high-grade, compact spectrometers for Earth Observation from SmallSats, several Dutch space companies decided to join TNO and establish a consortium that intends to provide 'endto-end' Earth Observation solutions with nano- and microsatellites, aiming at the production of small series. In the long run the portfolio of the consortium will include:

- Mission development

- Instrument/platform design

- Data retrieval/processing

- Launch arrangement
- Ground segment

- Modelling/data assimilation

- Customized data service

Currently the Spectrolite consortium consists of the following organizations: Airbus Defence \& Space Netherlands, Innovative Solutions in Space (ISIS), Science \& Technology (S\&T) and TNO.

As a first potential business case for a SmallSat mission, we are looking into the monitoring of air quality over densely populated areas worldwide. Currently more than half the world's population is living in cities and this number will continue to increase in future. Thus, municipalities are confronted more and more with the challenge of dealing with complex issues, such as extreme weather, mobility and transport, energy supply, safety, environmental pollution on the one hand, and creating and maintaining a agreeable and healthy living environment on the other. And with regard to the latter, air quality plays a crucial role.

We intend to focus this first SmallSat mission on monitoring $\mathrm{NO}_{2}$ at high spatial resolution and multiple times per day. $\mathrm{NO}_{2}$ is a relevant marker of anthropogenic air pollution due to sources like transport, central heating as well as many different industrial activities and has a strong effect on human health. However, in urban areas $\mathrm{NO}_{2}$ is marked by its highly local and dynamic character, which exhibits strong gradients over relatively small areas. In order to pinpoint local sources and characterize their source signatures better, we propose to supply high-res $\mathrm{NO}_{2}$ data to a variety of cities/regions/countries multiple times per day using a constellation of nano- or microsatellites. An additional advantage of such a constellation, the cost of which can be shared by multiple end-users, is that it provides consistency in the air quality measurements as well as large regional coverage.

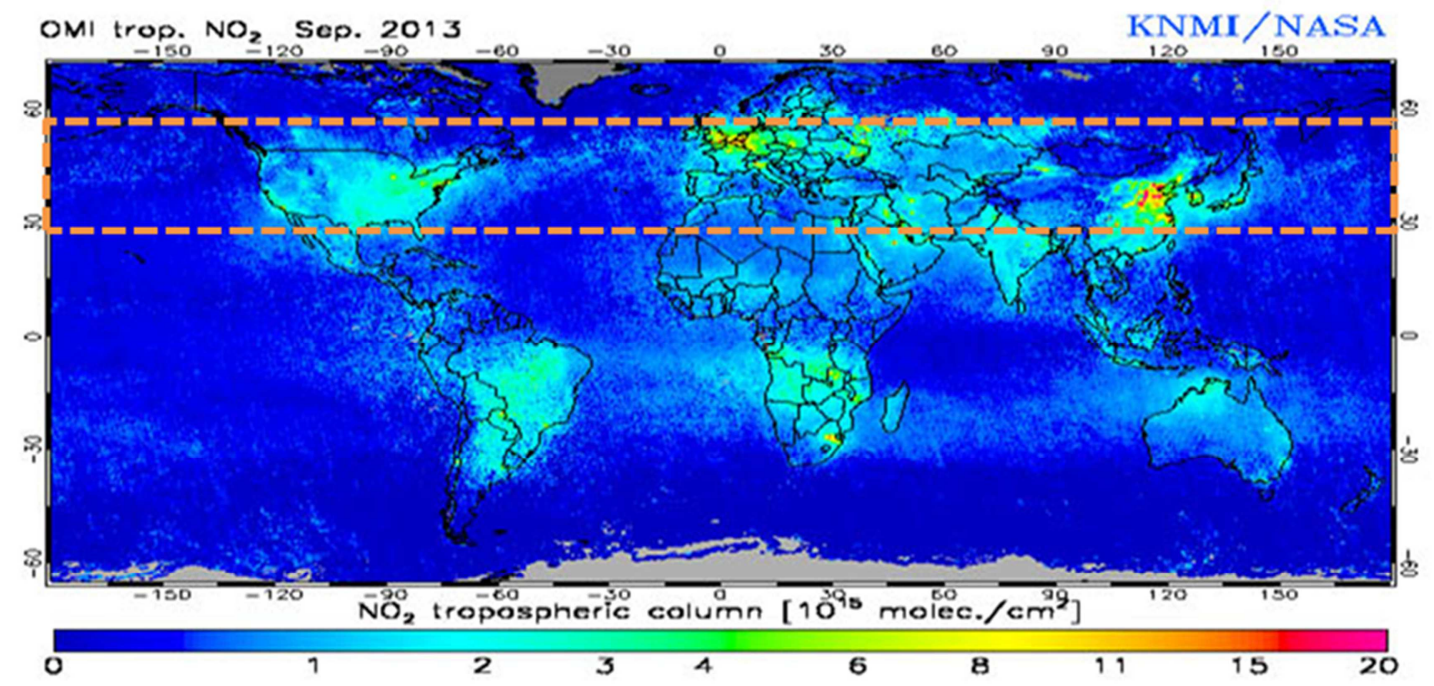

Figure 12. Map of the monthly $\mathrm{NO}_{2}$ concentration worldwide (Sep 2013); dashed lines show our region of interest covering almost all major cities in the Northern hemisphere (source: KNMI/NASA). 
Figure 12 shows a potential mission scenario where a constellation of small satellites covers the Northern hemisphere between $29^{\circ}$ Northern latitude and $56^{\circ}$ Northern latitude. This region is selected to be able to supply data on almost all major cities on the Northern hemisphere $\left(29^{\circ} \mathrm{N}\right.$ : Houston, Cairo and Chongdu and $56^{\circ} \mathrm{N}$ : Edinburgh and Moscow). We performed an analysis to compare the performance of different constellation configurations. Two constellations were selected for further analysis as shown in Figure 13: A constellation consisting of 9 satellites in three different SunSynchronous Orbits (SSO, @ $600 \mathrm{~km}, \sim 98^{\circ}$ incl.) and a constellation consisting of 9 satellites in three different lower inclination orbits (@600 km,60 incl.). The low-inclination orbit provides roughly twice the number of daily observations compared to the sun-synchronous constellation for a $56^{\circ}$ latitude location. This advantage decreases for lower latitudes. At $29^{\circ}$ latitude, the other end of our region of interest, both constellations provided about 3 observations per day. As the local observation time of a $60^{\circ}$ inclined orbit shifts roughly 20 minutes per day, it provides a good temporal sampling with a limited number of satellites. More information on this mission scenario can be found in ${ }^{4}$.
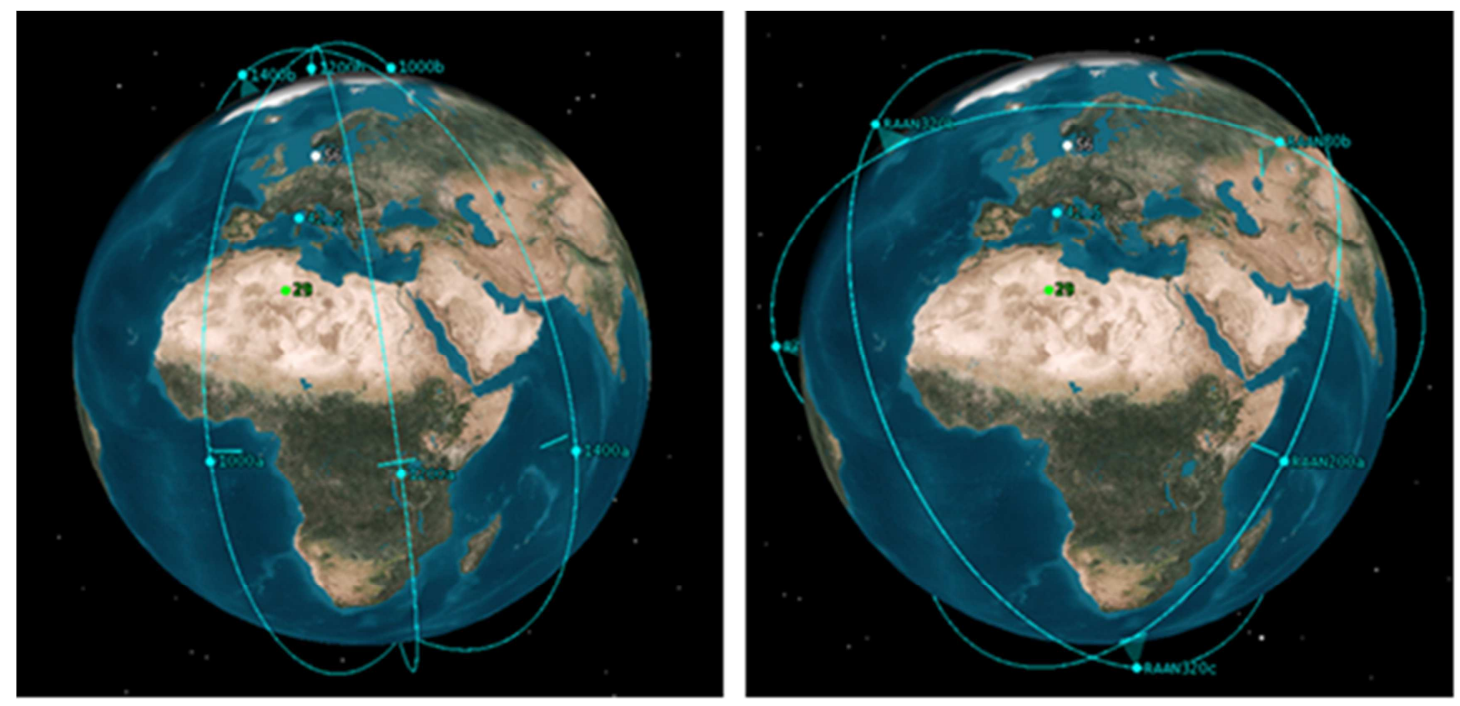

Figure 13. Two different satellite constellation configurations were considered; the left image shows three sun-synchronous orbits, the right image shows three $60^{\circ}$ inclined orbits.

\section{DISCUSSION AND CONCLUSIONS}

We have proposed and realized different innovative designs to manufacture compact, high-grade spectrometers for space applications in a cost-effective manner. To demonstrate the feasibility of these designs, we realised the Spectrolite breadboard. Spectrolite is based on an all-reflective, off-axis design, consisting of only 8 optical components, including 4 freeform mirrors and a flat grating in the spectrometer. This all-reflective approach has the following advantages:

- A favourable thermal stability due to the all-aluminium design (important for a low-mass satellite);

- The design can be copied unchanged for other wavelength ranges between $270 \mathrm{~nm}$ and $2400 \mathrm{~nm}$ (with the exception of the grating and possibly the detector);

- Fewer optical elements due to the insensitivity to chromatic aberrations and the application of diamond turned freeform optics;

- More design freedom regarding stray light baffling due to the off-axis nature of such a design;

- Inherently low ghost stray light between detector and optical surfaces. 
The Spectrolite housing was manufactured using a relatively novel method for space hardware: 3D printing and investment casting. The spatial accuracy of this process is in the order of $0.1 \mathrm{~mm}$, which is not sufficient if one wants to mount the mirrors directly without alignment. Therefore the mirror interfaces were post machined to an accuracy of 20 micron. The combination of 3D printing of the wax model and investment casting provides a large design freedom. The 3D printing of the wax model allows the casting to have a very complex shape, while the casting itself allows the design of accurate, lightweight and stiff structures.

Although the first Spectrolite castings showed only a few minor defects, first time right casting for geometric complex shapes is not 'business as usual'. Optimization in two or three casting designs should be taken into account. When producing multiple housings in small series, 3D printing + investment casting becomes more cost-effective than traditional milling. Cost savings of more than $20 \%$ can be achieved for series of 10 or more.

Although not substantiated in this paper, we are convinced that added manufacturing technologies will also require new design approaches, design approaches that will make optimal use of the new possibilities offered by these manufacturing technologies.

The manufacturing of the freeform mirrors turned out to be more challenging than expected, due to the more difficult mounting and the 'aggressive' shape with large slopes. This required a very low turning speed, which in turn required very high thermal stability of the cutting tool $(<10 \mathrm{~nm}$ over a period of minutes or more). The mirrors and the grating were assembled on manufacturing tolerance, which took approximately 1.5 days. The alignment of the detector was done in approximately one day. The spectral resolution varies between $\sim 0.2 \mathrm{~nm}$ and $\sim 0.33 \mathrm{~nm}$ and is best for the highest wavelengths.

By realizing the Spectrolite breadboard, we have successfully demonstrated the feasibility to design and manufacture very compact, high-grade spectrometers in a cost-effective manner. Given their size and weight these spectrometers can be flown on very small platforms, e.g. nano- and microsatellites. Moreover, they can easily be 'tuned' to observe different phenomena (e.g. air quality, land use or water quality) without requiring full redevelopment and qualification. This offers the opportunity to build these spectrometers in small series and share the development among multiple modules.

Initiated by the positive results of our efforts to design high-grade, compact spectrometers for Earth Observation from SmallSats, a number of Dutch space companies decided to join TNO and establish a consortium that intends to provide 'end-to-end' Earth Observation solutions with nano- and microsatellites, aiming at the production of small series.

The consortium will first focus on a SmallSat mission to monitor $\mathrm{NO}_{2}$ over densely populated areas. In order to pinpoint local sources and characterize their source signatures better, the consortium intends to supply $\mathrm{NO}_{2}$ data at high spatial resolution to a variety of cities/regions/countries multiple times per day using a constellation of nano- or microsatellites.

\section{REFERENCES}

[1] Doncaster B. and Shulman J., "2016 Nano/Microsatellite Market Forecast", published by SpaceWorks Enterprises Inc., Atlanta, USA, 2016.

[2] Volatier J-P., Baümer S., Kruizinga B. and Vink R., “Case study: TROPOLITE”, Proc. SPIE 9131, 91310L, May 1, 2014.

[3] Oosterling J.A.J., “Advanced Manufacturing Methods for Systems-of-Microsystems Nanospacecraft”, TNO technical note for ESA, ref. TNO-ESA-AM-00, 2015.

[4] Goeij B.T.G. de, Groot Z. de, Rotteveel J., Valk N. van der and Otter G., "Using small satellites and instruments for an operational air quality service", Proc. $10^{\text {th }}$ Symposium on Small Satellites for Earth Observation, Berlin, 2015. 